\title{
Guidelines are essential to improving clinical outcomes in breast cancer patients
}

\author{
Alberto J. Montero ${ }^{1}$
}

Published online: 6 August 2015

(c) Springer Science+Business Media New York 2015

Among the estimated \$3.08 trillion (18\% US GDP) [1], we will have spent this year in the U.S. on healthcare, a great deal of this is due to what is termed "unexplained clinical variation" [2]. This great variation of care is a major obstacle to improving quality and safety in health care, therefore finding ways to reduce it are necessary to successfully bend the cost curve in health care. While oncology encompasses a small fraction of the overall health costs, it represents one of the most rapidly increasing segments [3]. The Institute for Health Improvement has articulated a framework, known as the "triple aim" to reduce clinical variation and help optimize health system performance [4]. In order to make necessary changes to our current health care system, three distinct but interrelated areas need to be simultaneously addressed. These are as follows: (i) improve the health of populations; (ii) improve the patient experience of care (including quality and satisfaction); and, (iii) reducing the per capita cost of health care.

Promulgation of evidence-based guidelines or pathways to treat cancer patients, which themselves are distilled from the best scientifically available evidence, is perhaps one of the most important strategies to address excessive variability in cancer care. In other words, if as physicians we consistently treat our patients according to practice guidelines - to use to term coined by Dr. Sackett "evidence based medicine"- this would be an efficient strategy to directly address the goals of the triple aim [5].

In this issue, Wolters et al. [6] present a retrospective study that asks an important question: do breast cancer

Alberto J. Montero

montero2@ccf.org

1 Department of Hematology Oncology, 9500 Euclid Avenue/ R35, Cleveland, OH 44195, USA patients treated according to national practice guidelines have better clinical outcomes than those who weren't? In this study, the authors evaluated differences in clinical outcomes between women undergoing adjuvant treatment for breast cancer whose treatment adhered $100 \%$ to German national practice guidelines, and women whose treatments plans did not conform $100 \%$ to these guidelines. The different therapeutic modalities evaluated here included surgical therapy, radiotherapy, adjuvant endocrine therapy, and adjuvant chemotherapy.

This analysis was done separately for women treated before and after the year 2000. The authors compared outcomes between two different time intervals 1991-2000 (TI1) and 2001-2009 (TI2). In these subgroups, the risk of recurrence (RFS) and overall survival (OS) were compared between patients whose treatment was either conformed $100 \%$ to national practice guidelines, or non-guideline conforming. Interestingly, the authors found that in women who received $100 \%$ guideline-conforming therapy, both OS and RFS were significantly improved compared with patients who were not treated in accordance with clinical guidelines (RFS: $p<0.001, \quad \mathrm{HR}=0.51, \quad 95 \% \quad \mathrm{CI}$ $0.44-0.59 ; \quad$ OS: $p<0.001, \quad \mathrm{HR}=0.46, \quad 95 \% \quad$ CI $0.40-0.53)$. They also reported that clinical outcomes from TI1 to TI2 improved even for patients whose treatment was non-guideline adherent. The authors then conclude from this that clinical guidelines are not clinically "advantageous" in improving clinical outcomes among breast cancer patients. However, in quality terms, the data presented in this paper show quite convincingly that at least in patients treated $100 \%$ according to national guidelines clinical outcomes did improve, presumably though a reduction in the variation of care. Patients treated on pathway did better than those who were not. The fact that even breast cancer patients whose care was not $100 \%$ 
adherent to evidence-based practice guidelines had measurable improvements in clinical outcomes does not mean that guidelines are ineffective. Quite the contrary, this improvement in outcomes occurred precisely because of advances in breast cancer care. The authors used an impossibly high standard of adherence-anything short of $100 \%$ adherence to evidence-based guidelines was considered non-adherent in this analysis. That means, even in patients that were treated in a manner that was $80 \%$ adherent with evidence-based guidelines their treatment was classified as non-adherent. So it is not surprising then that even patients in the non-adherent group did better over time, because most of these patients were likely treated in some degree according to evidence-based guidelines. Even if they were not treated $100 \%$ according to practice guidelines, some of their care was likely guideline adherent.

A $100 \%$ adherence standard for clinical guidelines is an impossible standard to expect from both patients and clinicians. Too much adherence to guidelines is not necessarily good, and would stifle medical innovation. Some other reasonable level of adherence to medical guidelines should be expected.

One of the major unanswered questions of this study was that it did not explore why breast cancer patients were not treated according to national guidelines by their oncologists. In other words, what were the factors behind non-adherence? Were there any discernible patterns on the doctor side as to who was not practicing according to national guidelines? Implementation and dissemination of evidence-based guidelines into routine practice is difficult, due to a variety of many different factors which are well described in the literature [7-11].

Therefore, understanding why certain guidelines were or were not followed would ideally help to build better systems to enhance adherence among physicians. Finally, when it comes to following evidence-based guidelines, physicians are only half of the equation. We all know that many times the best available evidence may not apply to an individual patient. There are also many patient-driven factors which clearly can have a major impact on adherence to evidence-based guidelines, e.g., socio-economic factors, beliefs, and preferences. Regardless of the reasons-patient or physician related-for non-adherence to guidelines, this study demonstrated rather convincingly that clinical outcomes of breast cancer patients were significantly improved by practicing evidence-based medicine. And, as the U.S. health care system rapidly accelerates toward a pay for performance model, studies like this one are evidence that we are moving in the right direction.

\section{References}

1. Keehan SP, Cuckler GA, Sisko AM, Madison AJ, Smith SD, Wolfe CJ, Stone DA, Poisal JA, Lizonitz JM (2015) National health expenditure projections, 2014-24: spending growth faster than recent trends. Health Aff. doi:10.1377/hlthaff.2015.0600

2. Grossbart SR, Agrawal J (2012) Conceptualization and definitions of quality. In: Nash DB, Clarke J, Skoufalos A, Horowitz M (eds) Health care quality: the clinician's primer. ACPE, Sydney Olympic Park

3. Schnipper LE, Davidson NE, Wollins DS, Tyne C, Blayney DW, Blum D, Dicker AP, Ganz PA, Hoverman JR, Langdon R et al (2015) American Society of Clinical Oncology statement: a conceptual framework to assess the value of cancer treatment options. J Clin Oncol 33(4):901-910

4. Berwick DM, Nolan TW, Whittington J (2008) The triple aim: care, health, and cost. Health Aff 27(3):759-769

5. Sackett DL (1997) Evidence-based medicine: how to practice and teach EBM. Churchill Livingstone, New York

6. Wolters R, Wischhusen J, Stüber T, Weiss CR, Krockberger M, Bartmann C, Blettner M, Janni W, Kreienberg R, Schwentner L, Novopashenny I, Wischnewsky M, Wöckel A, Diessner J (2015) Guidelines are advantageous, though not essential for improved survival among breast cancer patients. Breast Cancer Res Treat 152(2):357-366

7. Cabana MD, Rand CS, Powe NR, Wu AW, Wilson MH, Abboud PA, Rubin HR (1999) Why don't physicians follow clinical practice guidelines? A framework for improvement. JAMA 282(15):1458-1465

8. Grol R (2001) Successes and failures in the implementation of evidence-based guidelines for clinical practice. Med Care 39(8 Suppl 2):II46-54

9. Grimshaw J, Eccles M, Tetroe J (2004) Implementing clinical guidelines: current evidence and future implications. J Contin Educ Health Prof 24(1):S31-37

10. Grimshaw JM, Shirran L, Thomas R, Mowatt G, Fraser C, Bero L, Grilli R, Harvey E, Oxman A, O'Brien MA (2001) Changing provider behavior: an overview of systematic reviews of interventions. Med Care 39(8 Suppl 2):II2-45

11. Francke AL, Smit MC, de Veer AJ, Mistiaen P (2008) Factors influencing the implementation of clinical guidelines for health care professionals: a systematic meta-review. BMC Med Inform Decis Making 8:38 